\title{
A Physics-based prognostics approach for Tidal Turbines
}

\author{
Fraser Ewing \\ Industrial Doctoral Centre for \\ Offshore Renewable Energy \\ University of Edinburgh \\ Edinburgh, U.K \\ f.ewing@ed.ac.uk
}

\author{
Philipp R. Thies \\ Senior Lecturer, Renewable \\ Energy \\ University of Exeter \\ Penryn, U.K \\ p.r.thies@exeter.ac.uk
}

\author{
Jonathan K. Shek \\ Chancellors Fellow, School of \\ Engineering \\ University of Edinburgh \\ Edinburgh, U.K \\ j.shek@ed.ac.uk
}

\author{
Claudio Bittencourt Ferreira \\ Business Development Director, \\ Renewables Certification \\ DNV GL Energy, London U.K \\ claudio.bittencourt.ferreira@dnv \\ gl.com
}

\begin{abstract}
Tidal Stream Turbines (TST) have the potential to become an important part of the sustainable energy mix. One of the main hurdles to commercialization is the reliability of the turbine components. Literature from the Offshore Wind sector has shown that the drive train and particularly the Pitch System (PS) are areas of frequent failures and downtime. The Tidal energy sector has much higher device reliability requirements than the wind industry because of the inaccessibility of the turbines. For Tidal energy to become commercially viable it is therefore crucial to make accurate reliability assessments to assist component design choices and to inform maintenance strategy. This paper presents a physics-based prognostics approach for the reliability assessment of Tidal Stream Turbines (TST) during operation. Measured tidal flow data is fed into a turbine hydrodynamic model to generate a synthetic loading regime which is then used in a Physics of Failure model to predict component Remaining Useful Life (RUL). The approach is demonstrated for the failure critical Pitch System (PS) bearing unit of a notional horizontal axis TST. It is anticipated that the approach developed here will enable device/project developers, technical consultants and third party certifiers to undertake robust reliability assessments both during turbine design and operational stages.
\end{abstract}

Keywords- Tidal Turbines, Physics-based Prognostics, Reliability, Fatigue, RUL

\section{INTRODUCTION}

The potential of Tidal stream energy to be part of a diversified carbon free generation mix is recognised globally. However, one of the central challenges facing the industry is device reliability [1]. Both the European Commission and the United States Bureau of Ocean Energy Management have stated that ensuring the reliable operation of Tidal Stream Turbines (TST's) is crucial if the industry is to become commercially viable. Improvements in component reliability and hence reductions in Operations \& Maintenance $(\mathrm{O} \& \mathrm{M})$ costs can be realised with the aid of Prognostics and Health Management (PHM) techniques.

Physics based prognostic approaches use a physical model to describe the degradation behaviour of a component and incorporate measured data and usage conditions to determine the model parameters. These models are advantageous because they are intuitive and based on physical phenomena and are not computationally expensive [2]. However, they are highly component specific and care must be taken when using them because other failure driving mechanisms may inadvertently be neglected. Data driven approaches utilise historical sensor data to identify characteristics of degradation and predict future degradation trend. This approach requires a large quantity of sensor data that is difficult to come by in a nascent industry such as Tidal stream energy.

The ocean current energy industry is currently in the precommercial stage. Tidal barrages have been used for many years to extract energy from the vertical motion of the ocean, however they are less popular in modern day society because of their large impact on the local environment and their large capital costs [3]. TST have the potential to mitigate these issues by deploying out in the open ocean and having minimal impact on the environment (they don't require large civil structures to house the turbines). TST utilise the horizontal flow of ocean currents and convert this into electrical energy, with a power take off (PTO) arrangement akin to modern wind turbines. The appeal of tidal stream energy is partly because of the 'predictable' nature of the tides. The harmonic constituents of the tidal currents for a geographical location can be determined and quantified over millennia. This means that the resource is much more predictable than for wind energy and could potentially provide baseload power and displace traditional methods such as nuclear [4]. The main locations that have been earmarked for large scale tidal stream development are the Pentland Firth (United Kingdom), Bay of Fundy (Canada), Lombok Straights (Indonesia) and Alderney Race (France).

This paper proposes a physics based prognostics approach for the reliability assessment of Tidal turbines during operation. A physics-based approach is beneficial as long term reliability predictions are more accurate than when using data driven techniques [2]. The lack of available historical sensor data also rules out data driven methods. The prognostics approach outlined here involves the continuous updating of a Physics of Failure (PoF) model with loading information. Tidal flow measurements from an Acoustic Doppler Current Profiler (ADCP) located at a site in the Pentland Firth, Scotland (Fig. 1) are used to generate representative daily flow profiles for the turbine hydrodynamic model. The hydrodynamic model then generates the loading profiles experienced by the turbine for 
each day. The PoF model calculates the bearing RUL based on the loading history experienced (inclusive of all days up to the current point in time). This approach enables the RUL estimate to be continuously refined as more data is fed into the model. The approach is demonstrated for the failure critical PS bearing unit.

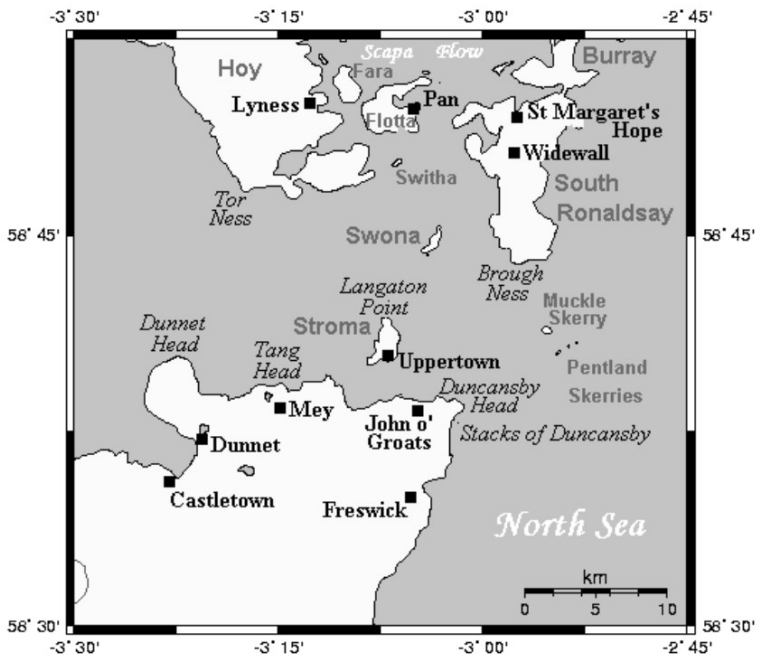

Fig. 1. Pentland Firth, Scotland

\section{THEORETICAL BACKGROUND}

\section{A. Tidal Theory}

The tides are a result of the movement of the Moon and Sun. Every 24 hours and 50 minutes the Moon orbits the Earth. This creates the largest gravitational force experienced by the Earth and results in the largest tidal (harmonic) constituent: the principal lunar semi diurnal (M2) [5]. One period of the M2 is approximately 12 hours and 25 minutes which results in a high and a low tide. The principal solar semidiurnal constituent (S2) is a result of the position of the Sun relative to the Moon; the tidal height is increased (spring tide) when the two bodies gravitational forces are parallel and then decreased (neap tide) when the forces are perpendicular. This means that the life a tidal turbine can be approximated using a two week flow profile (representing one spring and one neap cycle [6]). The tidal flow regime is dictated largely by gravity along with seabed bathymetry and weather conditions. Thus, flow profiles tend to be site specific and hence turbine operating characteristics from one site may be very different to those at another site.

The focus of this research is on the impact of the current flow and as such wave influence is neglected. As well as the velocity of the flow, turbulence is a key contributor to the tidal regime. Turbulent eddies are caused by the interaction of the flow with the seabed. The closer to the seabed the more retardation of the flow occurs. As the flow is slowed by the bed, higher up in the water column faster flowing water spills over the slower water resulting in a velocity gradient. A turbulent flow is in a continuously fluctuating state and thus in under to quantify it statistical descriptors are required [5]. The turbulence intensity (TI) is a commonly used descriptor:

$$
T I=\frac{u}{\sigma}
$$

Where $u$ is the mean velocity for a stationary time series of velocity measurements and $\sigma$ is the standard deviation of the measurements. The mean of a varying signal can be approximated with a variety of approaches (linear detrending was employed in this work as it is good at maintaining the integrity of the signal [7]).

\section{B. Hydrodynamic Modelling of Tidal Turbines}

The Tidal Turbine in this research is notional however it is based on typical early commercial turbines. The turbine has a drive train architecture akin to modern wind turbines which includes an electro-mechanical pitch system (PS), a permanent magnet generator (PMG), small epicyclic gearbox and a power converter. The rotor diameter is $18 \mathrm{~m}$ and the turbine is rated at $1.5 \mathrm{MW}$ at a flow speed of $3 \mathrm{~m} / \mathrm{s}$.

A Hydrodynamic model of the turbine is used to generate synthetic loading data. DNV GL's Tidal Bladed software is the modelling environment. It is a Blade Element Momentum Theory (BEMT) software that performs time domain hydrodynamic loading calculations to provide information to aid turbine design. The software has been through an extensive validation regime, details of which can be found here [8]. The comparison between the simulated and measured flapwise (overturning) bending moment at the blade root for the ALSTOM Deepen turbine can be seen in Fig. 2. There is a good agreement between the modelled moment $M_{y}$ and the strain gauge measurements. This provides a degree of certainty when using the hydrodynamic model to make predictions about turbine loading and performance. However, it is necessary to perform this validation exercise for each new turbine and location.

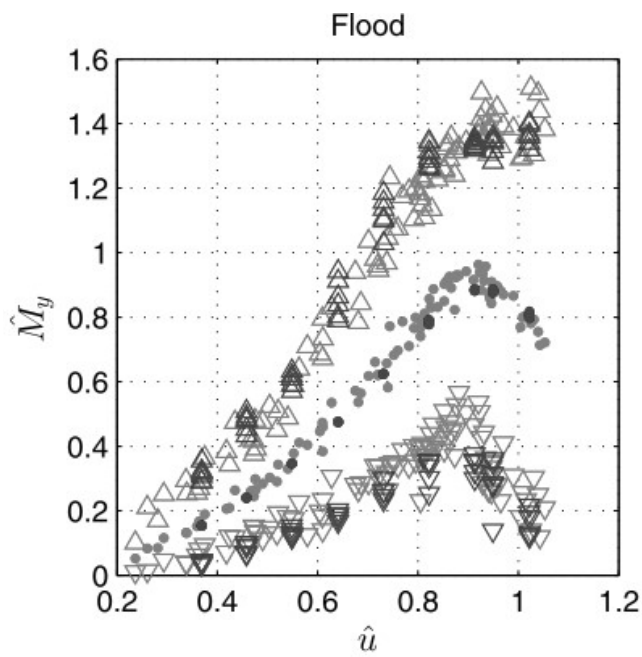

Fig. 2. Flapwise bending moment (My) vs. mean velocity for simulated (red) and measured (blue) data. Upward triangles are max, dots are mean and downward triangles are min (from [8])

\section{Physics of Failure for Tidal Turbines}

The PS is deemed to be one of the most failure critical components of horizontal axis turbines [9],[10]. One of the 
main parts of the PS is the bearing unit which exists at the root of each blade. Many studies have found that rolling contact fatigue at the bearing raceway is often the dominant failure mechanism in bearing units [11].

There are numerous approaches used to calculate bearing fatigue life. The ISO Standard 281 is widely regarded as one of the most robust [12]. This approach is based on the LundbergPalmgren theory that estimates rolling contact fatigue life [13]. The Lundberg-Palmgren model is associated with fatigue of the raceway surfaces and not the rolling elements (other methods can be used for this). Bearing life $L_{I S O m}$ is determined as a function of revolutions of the rotating raceway. The L10 describes a $10 \%$ probability of failure (or $90 \%$ probability of survival) for a bearing unit in terms of millions of revolutions of the rotating raceway (or in terms of hours of operation). In TST applications (as well as wind turbines) the bearing motion is oscillatory and the life model must be extended to accommodate this. The L10 life equation as per the ISO 281 method is:

$$
L_{I S O m}=a_{1} a_{I S O}\left(\frac{C_{a}}{P_{e a}}\right)^{p}
$$

Where the exponent $p$ is 3 for ball bearings and 10/3 for roller bearings. This exponent represents the slope of the fatigue curve and is dependent on material [14]. $a_{1}$ and $a_{I S O}$ are life modification and ISO factors respectively, $P_{e a}$ is the equivalent dynamic load on the bearing and $C_{a}$ is the basic dynamic axial load rating of the bearing (typically given in the manufacturers catalogue). For thrust roller bearings as used in this application $C_{a}$ can be calculated:

$$
C_{a}=f_{c m}(i \cos \alpha)^{7 / 9} Z^{3 / 4} D^{29 / 37} \tan \alpha
$$

Where $f_{c m}$ is a material factor based on CVD 52100 steel (as found in the ISO 281 standard), $\alpha$ is the nominal contact angle in degrees, $Z$ is the number of rolling elements per row and $D$ is the bearing outside diameter in millimeters.

$$
C_{a \_ \text {osc }}=C_{a}\left\{\frac{180}{\theta}\right\}^{3 / 10} Z^{0.033}
$$

Where $\theta$ is the equivalent oscillation amplitude (details of this calculation can be found in [15]). The important loadings that affect the bearing fatigue life are the overturning moment (flapwise), the axial force and the radial force. For a given load case, it is necessary to calculate the equivalent loads that are acting on the bearing unit. The equivalent load is the constant load that is equivalent to the time varying load. Based on the methodology in the National Renewable Energy Laboratory (NREL) Wind turbine Design Guideline for Pitch Rolling Bearings [12], the dynamic equivalent axial load for each load case $\left(P_{\text {eak }}\right)$ is calculated using:

$$
P_{\text {eak }}=0.75 F_{r}+F_{a}+\frac{2 M_{y}}{d_{p}}
$$

Where $d_{p}$ is the pitch diameter in millimeters. The equivalent constant load for each of the time varying forces and moments is approximated with the root mean square (RMS) value as per the NREL design guideline. By accounting for the duty cycle $\left(t_{k}\right)$, the oscillation speed $\left(N_{k}\right)$ and the oscillation amplitude $\left(\theta_{k}\right)$ each load case can be combined over each tidal cycle:

$$
P_{e a}=\left(\frac{\sum_{k=1}^{k=n} P_{e a k} N_{k} t_{k} \theta_{k}}{\sum_{k=1}^{k=n} N_{k} t_{k} \theta_{k}}\right)
$$

The oscillation amplitude $\left(\theta_{k}\right)$ for each load case is calculated via differencing of the time series of the simulated PS degree movement. The differencing method determines the relative movement from one time step to another. The mean value of oscillation amplitude $\left(\theta_{k}\right)$ is calculated for each stationary 10minute window. The oscillation speed $\left(N_{k}\right)$ is then calculated:

$$
N_{k}=\frac{\theta_{k}}{t_{k}}
$$

\section{Remaining Useful Life \& Reliability Metrics}

The fatigue life (L10 life) of the bearing unit is calculated after two flood and two ebb tide cycles on a continuous basis. The L10 is then used to calculate the RUL of the bearing:

$$
R U L=L_{I S O m}-T_{t}
$$

Where $T_{t}$ is the total PS operating time up to the current time $t$. As more data is input into the life model, the RUL estimate becomes more refined and more representative. The L10 value, representing the time at which the PS bearing has a $90 \%$ chance of being fully being operational can be converted into a probabilistic reliability function for the purposes of reliability assessment.

The Weibull distribution is typically used to describe the failure behavior of components due to its flexibility. With an estimation of the dimensionless shape parameter $\beta$ the Weibull distribution can be fully specified based on the calculated L10 value. The cumulative distribution function (CDF) or 'probability of failure' function for the Weibull distribution is given as:

$$
F(x, \beta, \eta)=1-e^{-\left(\frac{x}{\eta}\right)^{\beta}}
$$

Where $x$ is failure time in hours. The life parameter represents the time at which there is a $63.2 \%$ chance of failure. Assuming a $\beta$ parameter value of 1.5 [16] a straight line with gradient $\beta$ can be plotted on Weibull probability paper and used to calculate the life parameter $\eta$ (a horizontal line from the $63.2 \%$ ordinate point to the slope and then a vertical line down to the abscissa determines the $\eta$ value. The relationship between the two parameters has been determined for a range of $\beta$ values and can be seen in Fig. 3 . 


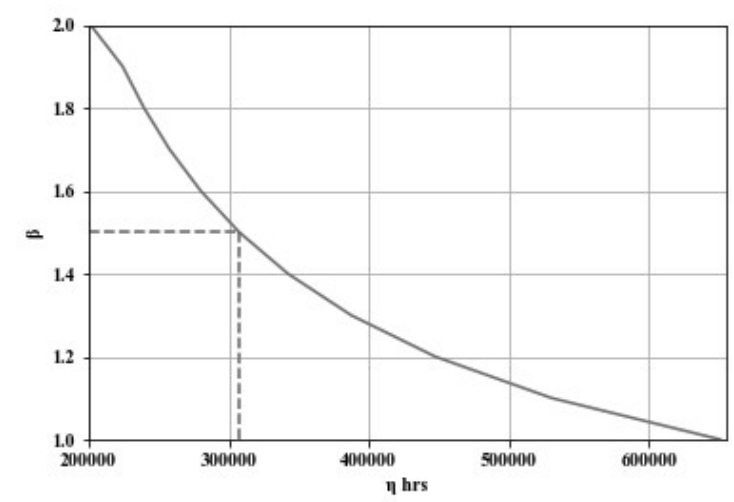

Fig. 3. Relationship between Weibull shape and life parameters. Red dotted line highlights $\eta$ value associated with $\beta$

\section{PRognostics CONCEPT}

Prognostics predicts the future damage or degradation and remaining useful life (RUL) based on measured data. Typically, the data comes from sensors that are measuring the damage criteria on board the turbine. This can be a direct measurement (such as capacitance levels in a battery) or indirect measurement (such as particle counters on a gearbox as a measure of gear wear). This sensor data can then be fed into a physical degradation model that relates the failure driver to some metric of usage e.g. time or cycles of operation. If no physical model exists and there is enough applicable historical data then statistical approaches can be used to learn this failure time/failure driver relationship. Given that the TST industry is currently in its nascent stage and there are few devices in the water, operational data is in short supply. However, this can be overcome by using a hydrodynamic model of the turbine to generate synthetic operational data. This synthetic data can then be run through Physics of Failure (PoF) models - in this case the bearing L10 model. In the future, as operational turbine sensor data becomes available, this can be incorporated into the process to calibrate the hydrodynamic model enabling more accurate RUL estimations. Calibration of the hydrodynamic model will also enable alterations to be made in a virtual environment (e.g. component design changes) and their effects on reliability assessed. After a long enough period (approx. 2 years) when sufficient operational data has accumulated, the synthetic data could be disregarded.

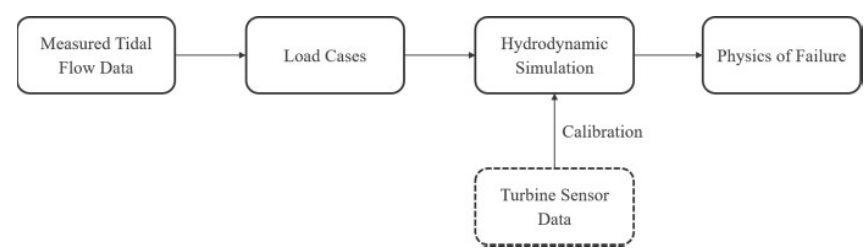

Fig. 4. Prognostics Methodology. Dashed Box highlights extension to current the model

The prognostics approach outlined in this research is suitable for use during turbine operation. The measured flow data is collected from an ADCP or equivalent measuring device located upstream of the turbine (to avoid interference from the turbine wake). This data is processed to generate tidal flow profiles that describe the velocity and turbulence. Hydrodynamic simulations are then run using the flow profiles. The simulated loadings on the component of interest are then input to a PoF model. After a period of two ebb and two flood tides (approx. 24 hours) the load cases are updated. This occurs repeatedly every 24 hours. The choice of 24 hours as the updating period is a trade-off between the accuracy of the load cases and the simulation run time. This approach can be used for different components and different failure mechanisms. Turbine sensor data (e.g. direct readings from the turbine rather than simulated) can be used to calibrate the hydrodynamic model as data becomes available. Rather than using the turbine sensor data directly in the PoF model it is beneficial to use the data for calibration such that the hydrodynamic model accuracy can be improved. If the hydrodynamic model can be calibrated to closely mirror the performance of the physical asset, then it can be used as a Digital Twin, enabling alterations such as component design changes to be made in a virtual environment.

\section{Pitch System BeARING CASE STUdy}

The prognostics concept is now demonstrated for the Pitch System bearing unit of a notional $1.5 \mathrm{MW}$ Tidal turbine. The same prognostics process can be followed for other components and failure mechanisms.

\section{A. Tidal Flow Profiles}

The first step in the approach involves the creation of representative flow profiles from the current flow measurements taken at the turbine site. The data is split into flood and ebb tides because the of the difference in turbulence intensity (TI) for each flow direction. The data is further split into 10-minute ensembles as this satisfies the condition of stationarity which must be applied when performing turbulence analysis [5]. The streamwise velocity is determined for each ensemble. The variation in the streamwise velocity over a two-week tidal cycle can be seen in Fig. 5 .
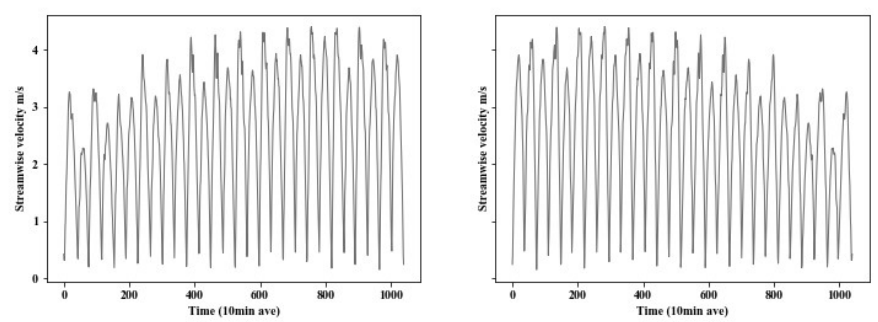

Fig. 5. Streamwise velocity variation for one spring and neap tidal cycle

The TI should be calculated for each 10minute ensemble however due to limitations in the data set average values of TI for Ebb (9\%) and Flood (12\%) tides are taken from a nearby site in the Pentland Firth [7].

\section{B. Load Cases}

Having extracted the necessary parameters from the tidal flow measurement data, the load cases can be generated. The load cases are updated after each 24-hour period (two flood and two ebb cycles). 

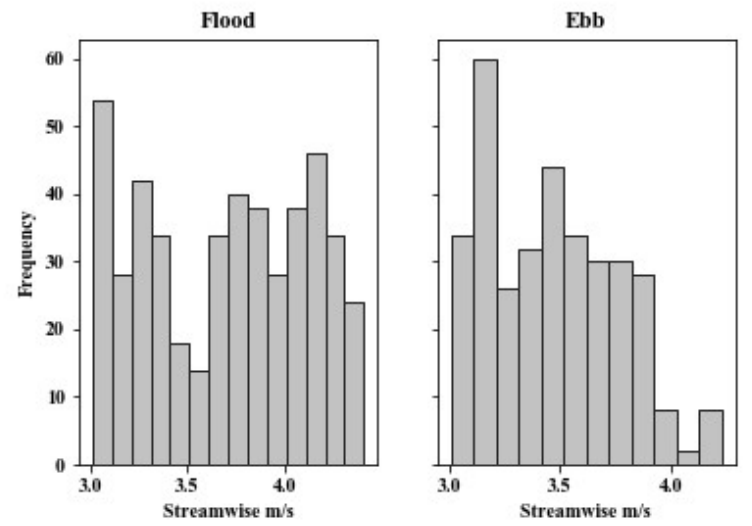

Fig. 6. Streamwise velocity profiles for Ebb and Flood tidal cycles after 4 days

An example load case table is demonstrated in Table I. In reality the TI\% would vary at different flow velocities however an average figure for each tidal cycle is used here. The Duty Cycle is another important parameter and represents the amount of time spent at each flow velocity.

TABLE I. LOAD CASES FOR FLOOD \& EBB TIDES AFTER TWO DAYS OPERATION

\begin{tabular}{|c|c|c|c|}
\hline $\begin{array}{c}\text { Streamwise hub } \\
\text { height velocity m/s }\end{array}$ & Tide & TI\% & Duty Cycle\% \\
\hline 3.06 & Flood & 12 & 21.8 \\
\hline 3.18 & Flood & 12 & 20.0 \\
\hline 3.29 & Flood & 12 & 14.5 \\
\hline 3.75 & Flood & 12 & 1.8 \\
\hline 3.86 & Flood & 12 & 5.5 \\
\hline 3.05 & Ebb & 9 & 9.1 \\
\hline 3.14 & Ebb & 9 & 16.4 \\
\hline
\end{tabular}

\section{Hydrodynamic Simulations}

The radial and axial forces and overturning moments are then resolved from the simulated root bending moments and forces for each load case. The overturning moment forces are the largest in magnitude and hence are the biggest contributors to the dynamic equivalent load that the bearing experiences (and thus the L10 value).

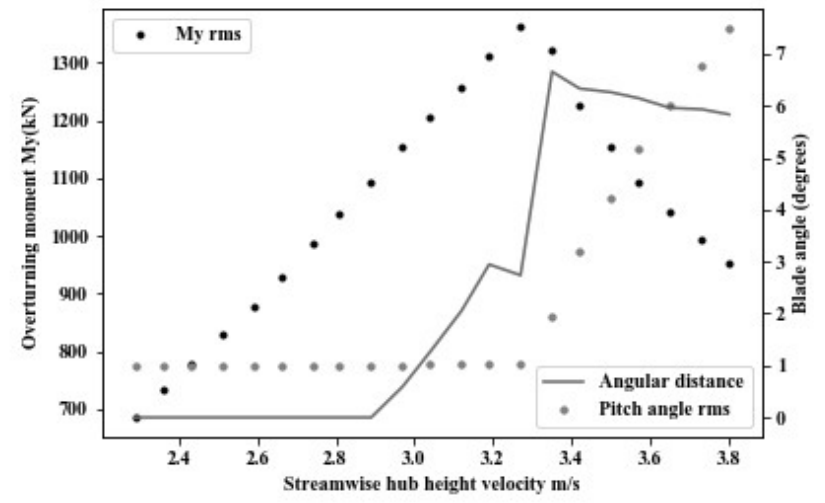

Fig. 7. Relationship between the overturning moment (My), pitch angle, pitch angular distance travelled and streamwise velocity.
The forces experienced by the bearing are affected by the operation of the PS controller as well as the tidal flow characteristics. As the turbine is rated at $1.5 \mathrm{MW}$ the controller comes into operation at a certain flow velocity to feather the blades, shed power and maintain the turbine power output at rated. Fig. 7 demonstrates the simulated operation of the PS controller across a range of streamwise velocities (with TI neglected). The largest overturning moments occur at lower flow velocities. This is because the PS controller causes the blades to pitch by a larger amount at higher flow velocities (to shed increasing amounts of power) which has the effect of reducing the moments experienced by the blades.

\section{Physics of Failure}

The bearing design parameters used in the bearing life model are listed in Table II. The hypothetical bearing is of the tapered roller variety. The bearing design is based on that of a prominent manufacturers specification as found in [17] and is suitable for a turbine with $18 \mathrm{~m}$ rotor diameter. One of the most influential bearing design parameters for fatigue life is the pitch diameter $d_{p}$. Increasing the pitch diameter has a direct reduction on the magnitude of the overturning moment.

TABLE II. ROLLER BEARING DESIGN PARAMETERS

\begin{tabular}{|l|c|c|}
\hline \multicolumn{1}{|c|}{ Design Parameters } & ID & Value \\
\hline Number of individual bearings & $\mathrm{Z}$ & 31 \\
\hline Diameter of individual bearings & $\mathrm{dw}$ & $45 \mathrm{~mm}$ \\
\hline Bearing contact angle & $\mathrm{alpha}$ & 45 \\
\hline Pitch diameter & $\mathrm{dp}$ & $849 \mathrm{~mm}$ \\
\hline Bearing Fatigue limit & $\mathrm{pu}$ & $800 \mathrm{kN}$ \\
\hline Axial Load Rating & $\mathrm{Ca}$ & $5.39 \mathrm{MN}$ \\
\hline
\end{tabular}

\section{E. Remaining Useful Life \& Reliability Metrics}

The L10 life of the bearing is continuously updated mon a daily basis across the two-week tidal cycle as can be seen in Fig. 8 . The estimates become more refined as data is included in the model. The variation in the bearing pitch diameter $d_{p}$ has been investigated holding all other bearing design parameters constant. This parameter is one of the most sensitive in the model and has a large effect on the overturning moment. A larger pitch diameter results in a larger bearing L10 life. As can be seen in Fig. 7 the largest overturning moments are experienced around the rated flow region. As the initial days' tidal flows are mainly concentrated around this region and because the overturning moment is the largest contributor to the fatigue life, the L10 estimate is low. Later as more days' data is added to the model the tidal profiles are not as concentrated around the rated flow region and as such the PS operates more to feather the blades and reduce the overturning moments. 


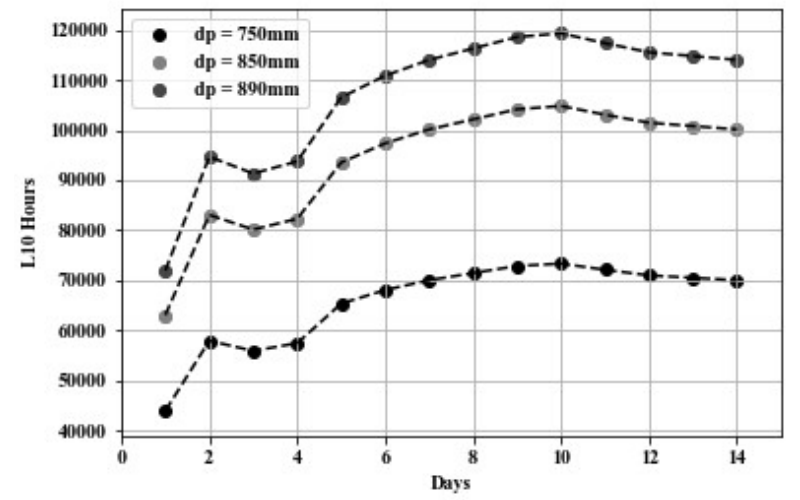

Fig. 8. Fatigue life estimate over the two-week tidal cycle for roller bearings with different pitch diameter $d_{p}$

Increasing the pitch diameter by $13 \%$ from $750 \mathrm{~mm}$ to $850 \mathrm{~mm}$ has the effect of increasing the L10 life by approx. $43 \%$. Increasing from $850 \mathrm{~mm}$ to $890 \mathrm{~mm}(4.7 \%)$ increases the L10 life by $11 \%$.

Reliability metrics such as the cumulative distribution function (CDF) which demonstrates the probability of failure can also be calculated. Translating the L10 into a reliability metric such as the CDF allows for the calculation of other reliability metrics such as failure rates which can then be used to inform design and maintenance strategy. As the L10 is specific to bearings, it is important to convert to a component agnostic metric such as the CDF to enable comparison with other components.

Table III shows the L10 values and the equivalent characteristic life values $(\eta)$ after each day's operation over the course of the two-week tidal cycle.

TABLE III. RELIABILITY METRICS FOR BEARING $d_{p}=850 \mathrm{MM}$

\begin{tabular}{|c|c|c|}
\hline & L10 hrs & $\boldsymbol{\eta} \boldsymbol{h r s}$ \\
\hline Day 1 & 63,046 & 281,667 \\
\hline Day 2 & 83,042 & 371,009 \\
\hline Day 3 & 80,112 & 357,919 \\
\hline Day 4 & 82,264 & 367,539 \\
\hline Day 5 & 93,509 & 417,782 \\
\hline Day 6 & 97,335 & 434,880 \\
\hline Day 7 & 100,108 & 447,266 \\
\hline Day 8 & 102,126 & 457,809 \\
\hline Day 9 & 104,140 & 466,837 \\
\hline Day 10 & 104,795 & 469,774 \\
\hline Day 11 & 103,055 & 461,974 \\
\hline Day 12 & 101,455 & 454,801 \\
\hline Day 13 & 100,786 & 451,802 \\
\hline Day 14 & 100,108 & 448,763 \\
\hline
\end{tabular}

The probability of failure estimate for the bearing unit during the first week of operation can be seen in Fig. 9. The refinement of the estimation is evident.

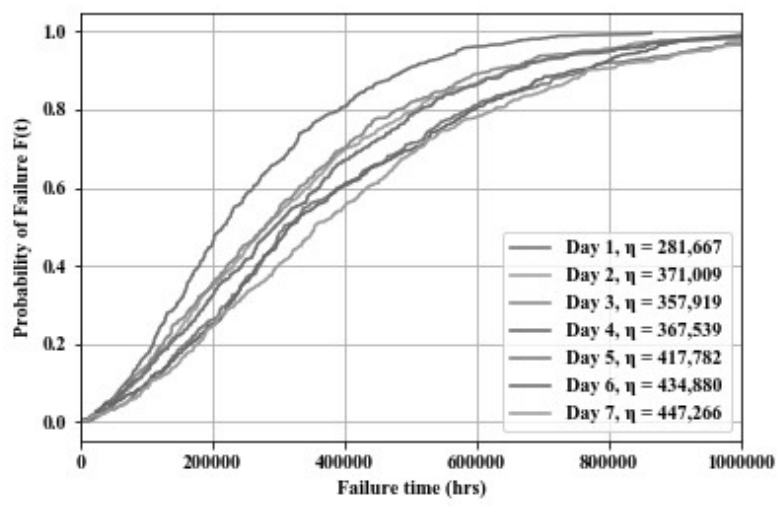

Fig. 9. Weibull failure probability function for the Pitch System bearing unit after over the first week of operation $\left(\beta=1.5, d_{p}=850 \mathrm{~mm}\right)$

\section{F. Calibration with sensor data}

As tidal turbine development proceeds and more devices become operational it is envisaged that turbine sensor data will become available and can then be incorporated into the prognostic methodology. Blade strain gauge data and PS sensor data can be used to calibrate the hydrodynamics model so that the performance of the model closely resembles that of the actual turbine, creating a 'Digital Twin'. For an acceptable calibration to take place, a minimum of 1 tidal cycle (twoweeks) operating data would be required.

\section{DISCUSSION}

The bearing life and reliability are sensitive to the tidal flow characteristics, the design parameters and the operation of the pitch system. The controller set point dictates at what flow velocity the pitch system operates and this directly influences the L10 life of the bearing. At regions close to the rated flow (i.e. near to the controller set point), the PS does not operate as much as when the flow velocity is larger. Therefore, the magnitude of the influential overturning moment $M_{y}$ is only slightly reduced when the flow velocity is near rated. When the flow increases to approx. $3.2 \mathrm{~m} / \mathrm{s}$ ( $8 \%$ above rated) the PS is operating continuously and as such the magnitude of $M_{y}$ is reduced.

The effect of changing the pitch diameter $d_{p}$ has also been explored. A larger $d_{p}$ has the effect of increasing the L10 life. This is because an increased bearing diameter results in a greater distribution of the forces acting on the bearing (rather than a higher concentration in a smaller area). A $43 \%$ increase in bearing fatigue life can be achieved by increasing the pitch diameter from $750 \mathrm{~mm}$ to $850 \mathrm{~mm}$. However, the effect of this design change on other components such as the blades must be considered. A larger pitch diameter would mean a larger diameter blade which would likely have a higher failure rate than a smaller diameter blade. It is important when conducting 
reliability assessments such as this to not neglect other components and failure mechanisms.

\section{CONCLUSIONS \& FURTHER WORK}

This paper has demonstrated a physics-based prognostics approach for the RUL estimation for the fatigue life of a Tidal Stream Turbine Pitch System bearing unit. Being a failure critical area and given a lack of operational data and very high reliability requirements, it is important for tidal stream turbine developers to be able to accurately quantify reliability and make informed RUL estimates.

The approach demonstrated here allows for a refinement of the life estimate of the component on a continuous basis, in keeping with typical prognostics methods. A continuous updating procedure is proposed that re-calculates the bearing life based on new loading information. This enables near real-time estimates of the RUL to be made. The method also demonstrates converting the L10 life metric into a component agnostic reliability metric such as the cumulative distribution function. This enables direct comparison between different components which is important for a nascent industry such as tidal energy in which turbine development is still going through iterative design.

Reliability has many drivers and although this paper has investigated one failure mechanism for one specific component, it is anticipated that more mechanisms can be incorporated and further failure critical components assessed. Also, the incorporation of operational turbine sensor data in the future will enable a calibration of the hydrodynamic model and more certainty in the life estimations. This extension would enable the operation of the hydrodynamic model as a Digital Twin in which the real turbine operation is mirrored in the virtual domain. This would be invaluable for the design process as the effect on the life of components by making design changes could be assessed in the virtual space.

It is anticipated that tidal turbine developers will benefit from the physics based prognostics approach developed here that enables RUL estimates in the absence of degradation data. Also, third party verifiers can use this approach to assess the designs of turbines as part of the certification process.

\section{ACKNOWLEDGMENTS}

The support of the ETI and RCUK Energy Program funding for IDCORE (EP/J500847/1) is gratefully acknowledged. The authors thank SIMEC Atlantis Energy Ltd for the provision of data and turbine models.

\section{REFERENCES}

[1] J. Wolfram, "On Assessing the Reliability and Availability of Marine Energy Converters: The Problems of a New Technology," Proc. Inst. Mech. Eng. Part O J. Risk Reliab., vol. 220, no. 1, pp.
55-68, 2006.

N.-H. Kim, D. An, and J.-H. Choi, Prognostics and Health Management of Engineering Systems. 2017.

[3] A. Roberts, B. Thomas, P. Sewell, Z. Khan, S. Balmain, and J. Gillman, "Current tidal power technologies and their suitability for applications in coastal and marine areas," J. Ocean Eng. Mar. Energy, vol. 2, no. 2, pp. 227-245, 2016.

[4] European Commission, Market study on ocean energy, no. May. 2018.

[5] W. E. Richard Thomson, Data Analysis Methods in Physical Oceanography. 2005.

[6] F. Elasha, D. Mba, M. Togneri, I. Masters, and J. A. Teixeira, "A hybrid prognostic methodology for tidal turbine gearboxes," Renew. Energy, vol. 114, pp. 1051-1061, 2017.

[7] B. Sellar and G. Wakelam, "Characterisation of Tidal Flows at the European Marine Energy Centre in the Absence of Ocean Waves," Energies, vol. 11, no. 1, p. 176, 2018.

[8] S. G. Parkinson and W. J. Collier, "Model validation of hydrodynamic loads and performance of a full-scale tidal turbine using Tidal Bladed," Int. J. Mar. Energy, vol. 16, pp. 279-297, 2016.

[9] A. J. Carroll, A. Mcdonald, and D. Mcmillan, "Offshore Wind Turbine and Sub-assembly Failure Rates through Time," 2015

[10] A. M. and D. M. James Carroll, "Failure rate, repair time and unscheduled O\&M cost analysis of offshore wind turbines," Wind Energy, vol. 17, no. April 2013, pp. 657-669, 2016.

[11] A. Cubillo, S. Perinpanayagam, and M. Esperon-Miguez, "A review of physics-based models in prognostics: Application to gears and bearings of rotating machinery," Adv. Mech. Eng., vol. 8, no. 8, pp. $1-21,2016$

[12] T. Harris, J. H. Rumbarger, and C. P. Butterfield, "Wind Turbine Design Guideline DG03 : Yaw and Pitch Rolling Bearing Life," Nrel, no. December, 2009.

[13] T. A. Harris and W. K. Yu, "Lundberg-Palmgren Fatigue Theory: Considerations of Failure Stress and Stressed Volume," J. Tribol., vol. 121 , no. 1 , p. $85,2008$.

[14] T. A. Harris and R. M. Barnsby, "Life ratings for ball and roller bearings," Proc. Inst. Mech. Eng. Part J J. Eng. Tribol., vol. 215, no. 6, pp. 577-595, 2001.

[15] International Organization for Standardization, "ISO 281:2007

Rolling bearings - Dynamic load ratings and rating life," vol. 3, no May, 2016.

[16] F. K. Bloch, Heinz P.; Geitner, Machinery Failure Analysis and Troubleshooting, Volume 2 - Practical Machinery Management for Process Plants (4th Edition) Appendix A. 2012.

[17] SKF, "SKF Rolling bearings Catalogue," 2012. 